\title{
Efficacy and safety of hybrid endoscopic submucosal dissection compared with endoscopic submucosal dissection for rectal neuroendocrine tumors and risk factors associated with incomplete endoscopic resection
}

\author{
Xiang-Yao Wang", Ning-Li Chai", En-Qiang Linghu, Hui-Kai Li, Ya-Qi Zhai, Xiu-Xue Feng, \\ Wen-Gang Zhang, Jia-Le Zou, Long-Song Li, Jing-Yuan Xiang \\ Department of Gastroenterology and Hepatology, Chinese PLA General Hospital, Beijing 100853, China \\ Contributions: (I) Conception and design: EQ Linghu, NL Chai, XY Wang; (II) Administrative support: EQ Linghu; (III) Provision of study \\ materials or patients: EQ Linghu, NL Chai, HK Li, YQ Zhai; (IV) Collection and assembly of data: XX Feng, WG Zhang; (V) Data analysis and \\ interpretation: JL Zou, LS Li, JY Xiang; (VI) Manuscript writing: All authors; (VII) Final approval of manuscript: All authors. \\ \#These authors contributed equally to this work. \\ Correspondence to: En-Qiang Linghu, MD, PhD. Department of Gastroenterology and Hepatology, Chinese PLA General Hospital, No. 28 Fuxing \\ Road, Beijing 100853, China. Email: linghuenqiang@vip.sina.com.
}

Background: Neuroendocrine tumors (NETs) are rising in prevalence, particularly with the rectal area. This study evaluated and compared the safety and effectiveness of hybrid endoscopic submucosal dissection (ESD) with those of ESD for rectal NETs and risk factors associated with incomplete endoscopic resection.

Methods: A total of 272 consecutive patients who underwent ESD or hybrid ESD for rectal NETs at the Chinese PLA General Hospital in the period from February 2011 to September 2018 were involved in this study. Data were collected from clinical and endoscopic databases. The procedure time, en bloc resection, complete resection, complication, and recurrence rates were evaluated.

Results: In the hybrid ESD group were 111 patients (who had 119 lesions between them), with a further 161 patients (164 lesions) in the ESD group. No significance was found in baseline characteristics between the two groups. Hybrid ESD had a significantly shorter mean procedure time than ESD $(13.2 \pm 8.3$ vs. 18.1 \pm 9.7 min, $\mathrm{P}=0.000)$. Hybrid ESD showed similar en bloc resection (99.2\% vs. $98.2 \% ; \mathrm{P}=0.373)$, complete resection $(94.1 \%$ vs. $90.9 \%, \mathrm{P}=0.641)$, and postprocedural bleeding $(2.5 \%$ vs. $0.6 \%, \mathrm{P}=0.313)$ rates to ESD. Univariate and multivariate analysis showed that higher histopathological grade was associated with incomplete resection.

Conclusions: For rectal NET, both ESD and hybrid ESD are effective and safe forms of treatment. Hybrid ESD provides an alternative option in the treatment of rectal NETs. Further developments are needed to improve the complete resection rate, especially concerning tumors with higher histopathological grade.

Keywords: Endoscopic submucosal dissection (ESD); hybrid endoscopic submucosal dissection (hybrid ESD); rectal neuroendocrine tumors (NETs); endoscopic resection (ER)

Submitted Oct 24, 2019. Accepted for publication Jan 14, 2020.

doi: $10.21037 /$ atm.2020.02.25

View this article at: http://dx.doi.org/10.21037/atm.2020.02.25 


\section{Introduction}

The prevalence of neuroendocrine tumors (NETs), particularly concerning the rectal area, is rising. The rectum is second only to the small intestine as a common location for digestive NETs (1). Rectal NETs have increased in incidence almost ten-fold over the past few decades, which is thought to be due to increased colorectal cancer screening, recent improvements in detection due to endoscopic developments, and greater clinical understanding (1-4). Rectal NETs can be asymptomatic and found incidentally, and despite rectal NETs generally being indolent, metastases can occur in some patients even with relatively small tumors (5). Hence, the early treatment and complete resection of rectal NETs are of great importance for achieving a good prognosis.

The management of rectal NETs depends on the tumor size, depth of invasion, and local lymphadenopathy status. Endoscopic resection (ER) is a safe and effective modality for treating small and localized tumors without adverse features or metastasis (6). Most rectal NETs can be removed by ER, such as conventional endoscopic mucosal resection (EMR) and modified EMR, but the outcomes of different ER techniques have varied in different studies. It has been reported that endoscopic submucosal dissection (ESD) has higher en bloc and complete resection rates than other endoscopic techniques, such as polypectomy and conventional EMR $(7,8)$. However, the process of ESD is relatively complicated and time consuming and can only be conducted by advanced endoscopists in tertiary centers. There is also a relatively high risk of perforation during the ESD procedure (9). Hybrid ESD, a simplified technique for ESD with snaring, has shown efficacy and safety similar to that of ESD for treating colorectal mucosal neoplasms, such as laterally spreading tumors $(10,11)$. However, there is a lack of studies on its use for treating rectal NETs. This study was designed to assess the safety and effectiveness of hybrid ESD in the treatment of rectal NETs and explore risk factors associated with incomplete resection.

\section{Methods}

\section{Patients and lesions}

Patients who had rectal NETs underwent ESD or hybrid ESD at the Chinese PLA General Hospital (Beijing, China) in the period between February 2011 and September 2018 were enrolled in this study. Data collected from a database were retrospectively analyzed, including patient and tumor characteristics, endoscopic procedures, and complications that had surfaced due to procedures. The exclusion criteria were as follows: (I) the histopathological result of the resected specimen was not NET or was mixed with another type of neoplasia; (II) muscularis invasion or lymph node or distant metastasis confirmed before the endoscopic procedure. Endoscopic ultrasonography (EUS) or abdominopelvic computed tomography (CT) were conducted to exclude muscularis invasion and lymph node or distant metastasis. Each patient was made fully aware of the benefits and risks related to the endoscopic intervention, and we obtained written informed consent before carrying out an endoscopic procedure. Approval was granted by the Ethics Committee of the Institutional Review Board of Chinese PLA General Hospital complying with the Declaration of Helsinki.

\section{Outcomes}

The outcomes of this study were to evaluate and compare procedure duration, en bloc resection, complete resection, complication, and recurrence rates of the two groups. Factors associated with short procedure time were analyzed using univariate and multivariate analysis. Univariate and multivariate analysis were also used to explore the factors related to incomplete resection.

\section{ESD and hybrid ESD procedures}

The patients with NETs were diagnosed by colonoscopy or were referred from other hospitals. We carried out EUS with the UM3R ultrasonic mini probe (UMP, $20 \mathrm{MHz}$; Olympus, Tokyo, Japan) before ER to evaluate the depth of the tumor invasion. A single-channel endoscope (GIFQ260J, PCF-Q260J, Olympus, Tokyo, Japan) tipped with transparent plastic was used for ESD and hybrid ESD. The VIO200D electrosurgical unit was used (ERBE, Tubingen, Germany). A flexible dual knife (Olympus, Tokyo, Japan) or argon plasma (ERBE, Tubingen, Germany) was used to mark the incision line, which was placed approximately $5 \mathrm{~mm}$ from the lesion periphery. Submucosal injection of a 1:10,000 epinephrine-normal saline solution combined with a small amount of methylene blue was performed on the area surrounding the lesion, providing a submucosal cushion. A dual knife or IT knife (Olympus, Tokyo, Japan) was used to perform the mucosal incisions, and submucosal dissection after the submucosal injection and the lesion and muscle layer were entirely separated from no snaring at all. 

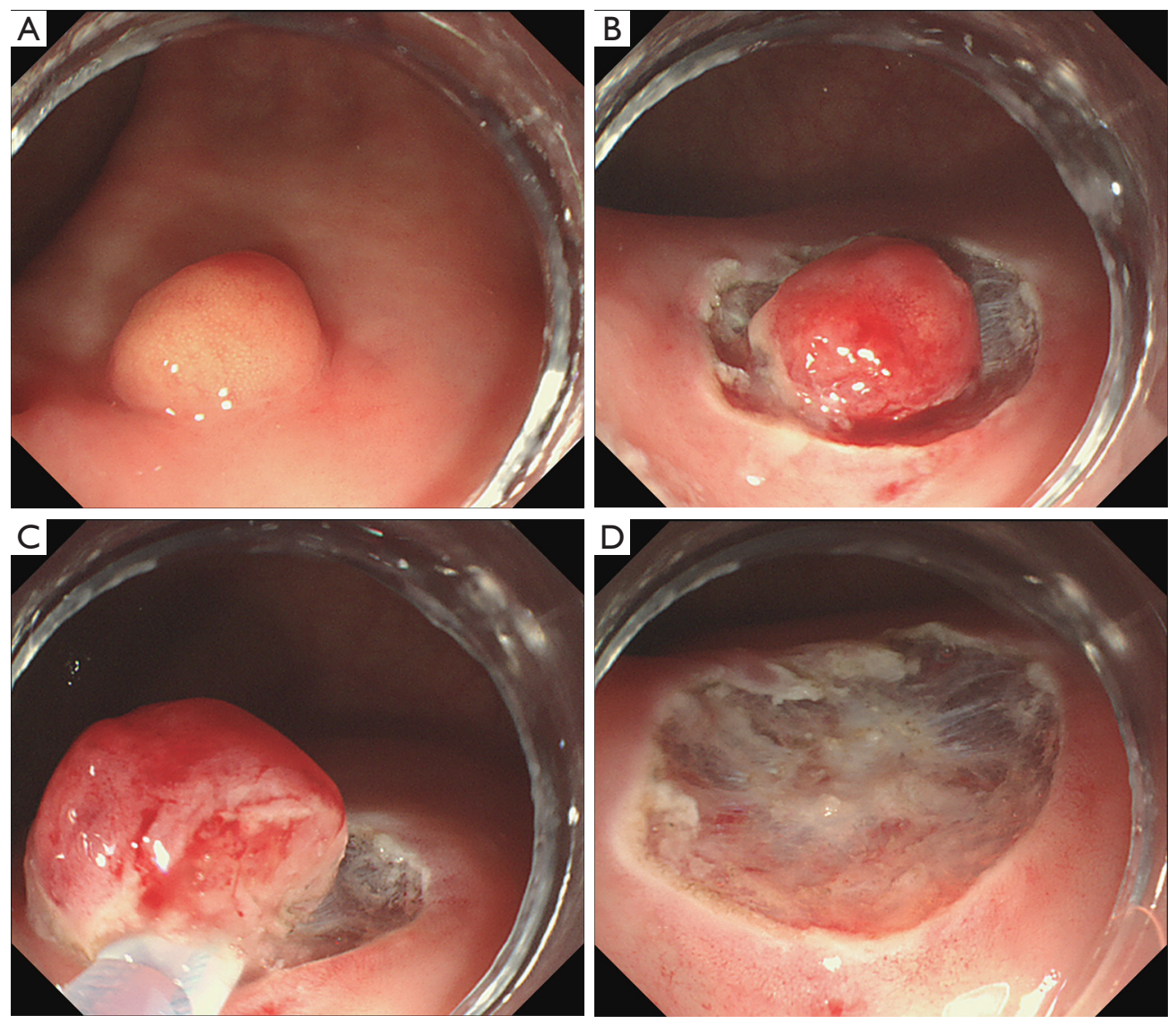

Figure 1 Hybrid endoscopic submucosal dissection (ESD) of a rectal neuroendocrine tumor (NET). (A) An approximately 10-mm rectal NET; (B) mucosal incision, and adequate submucosal dissection; (C) complete snaring resection; (D) a clear post-hybrid ESD defect.

Hemostatic forceps or argon plasma coagulation was used to stop bleeding during the procedure if necessary and to coagulate the visible vessels of the defect after resection to prevent delayed bleeding.

The hybrid ESD procedure was carried out in the following manner (Figures 1,2). The submucosal injection was performed along with the circumferential incision, as described above, for ESD. After an adequate amount of submucosal dissection, which should be performed at least to the bottom margin of the tumor, snaring was performed using a polypectomy snare (Cook, Winston-Salem, USA) to remove the undissected lesion completely. The other steps were similar to the ESD procedure.

The procedure time was measured from the submucosal injection until the ER was completed. Lesion size was measured through the use of open-biopsy forceps endoscopically, EUS or postoperative histopathological evaluation of the specimen.

\section{Histopathological evaluation}

Resected specimens were fixed to a specialized plate with a scale to measure the lesion size and were then fixed in formalin solution. The histopathological evaluation included the histological type, lateral and vertical resection margins, and invasion layer, which was determined by hematoxylin \& eosin (H\&E) and immunohistochemical (IHC) staining in line with the 2010 World Health Organization classification of tumors of the digestive system (12).

Endoscopic en bloc resection was defined as the resection of the entire tumor in a single piece, as observed endoscopically. Resection, which left the vertical and lateral margins free of neoplasia by histopathological evaluation, was considered to be complete resection. Incomplete resection was considered to have occurred when a resection failed to achieve tumor-free margins (13). 
A

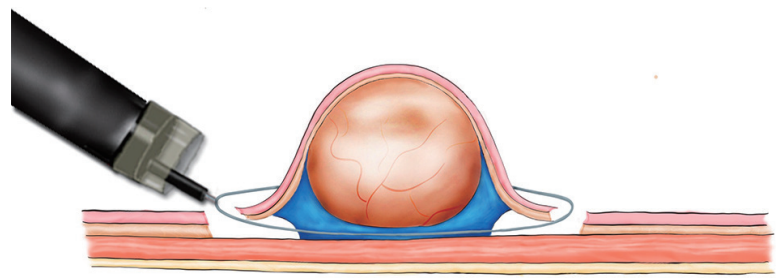

B

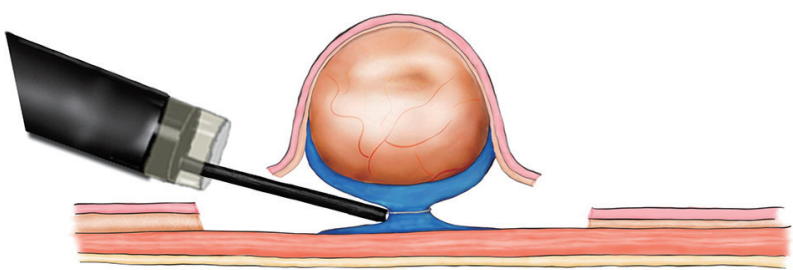

Figure 2 Schematic diagrams of hybrid endoscopic submucosal dissection (ESD) for rectal neuroendocrine tumors (NET). (A) Snare was placed after adequate submucosal dissection; (B) complete snaring resection was performed for the undissected part.

\section{Complications}

Postprocedural bleeding and perforation were two of the main procedure-related complications. Postprocedural bleeding was considered to have occurred when a patient experienced hematochezia, which required endoscopic hemostasis following the procedure. A visible hole in the rectal wall during the procedure was classed as perforation, as was the detection of free air by radiological examination in the time following the procedure.

\section{Follow-up}

Patients underwent scheduled follow-up endoscopic examinations. A pathologically confirmed diagnosis of NET in the same location for more than 6 months following the initial procedure was classed as local recurrence. Patients with complete resection were followed at 6,12 , and 24 months after the procedure and biennially after that if no recurrence was detected. For patients with incomplete resection who refused additional surgery, follow-up endoscopy was scheduled at 3, 6, and 12 months and yearly after that if no local recurrence was identified. A biopsy was performed if recurrence was suspected during the follow-up endoscopic examination. The existence of lymph node or distant metastasis was evaluated by abdominopelvic CT or US every 12 months.

\section{Statistical analysis}

Continuous variables are presented as the mean with standard deviation (SD) or the median with range and were evaluated by the $t$-test or the Mann-Whitney $\mathrm{U}$ test. Categorical data are expressed in the form of numbers and percentages, and analysis was conducted through a Chi- square test. Multivariable logistic regression analysis was conducted for factors relating to shorter procedure time and incomplete resection. Statistical significance was considered to exist when $\mathrm{P}<0.05$. SPSS statistical software version 23.0 (SPSS, Inc., Chicago, IL, USA) was used to conduct all data analyses.

\section{Results}

\section{Baseline characteristics and clinical outcomes}

Two hundred and seventy-six patients were eligible to undergo ESD or hybrid ESD in this study (Figure 3). Four patients did not qualify for inclusion due to negative histopathological results, hepatic metastasis before the procedure, and tumors mixed with laterally spreading tumors. In the ESD group were 161 (with 164 lesions), and in the hybrid ESD group were 111 patients (who had 119 lesions between them). Table 1 shows the patients' baseline characteristics. The mean lesion sizes of the hybrid ESD and ESD groups were $6.7 \pm 2.9$ and $7.1 \pm 3.6 \mathrm{~mm}$, respectively. There was only one patient in the ESD group with a lesion diameter of $30 \mathrm{~mm}$; the others were all less than $20 \mathrm{~mm}$. Ten patients with multiple lesions; one patient in the hybrid ESD group had three lesions, and the others had two lesions each.

Table 2 lists the outcomes of the resection of lesions by ESD and hybrid ESD. The procedure time in the hybrid group was significantly shorter than in the ESD group $(\mathrm{P}=0.000)$. The overall en bloc resection rate was $98.6 \%$, and the complete resection rate was $92.2 \%$; no significant differences were found between the two groups. Three patients who underwent hybrid ESD experienced postprocedural bleeding, as did one in the ESD group, and these patients received endoscopic hemostasis treatment 


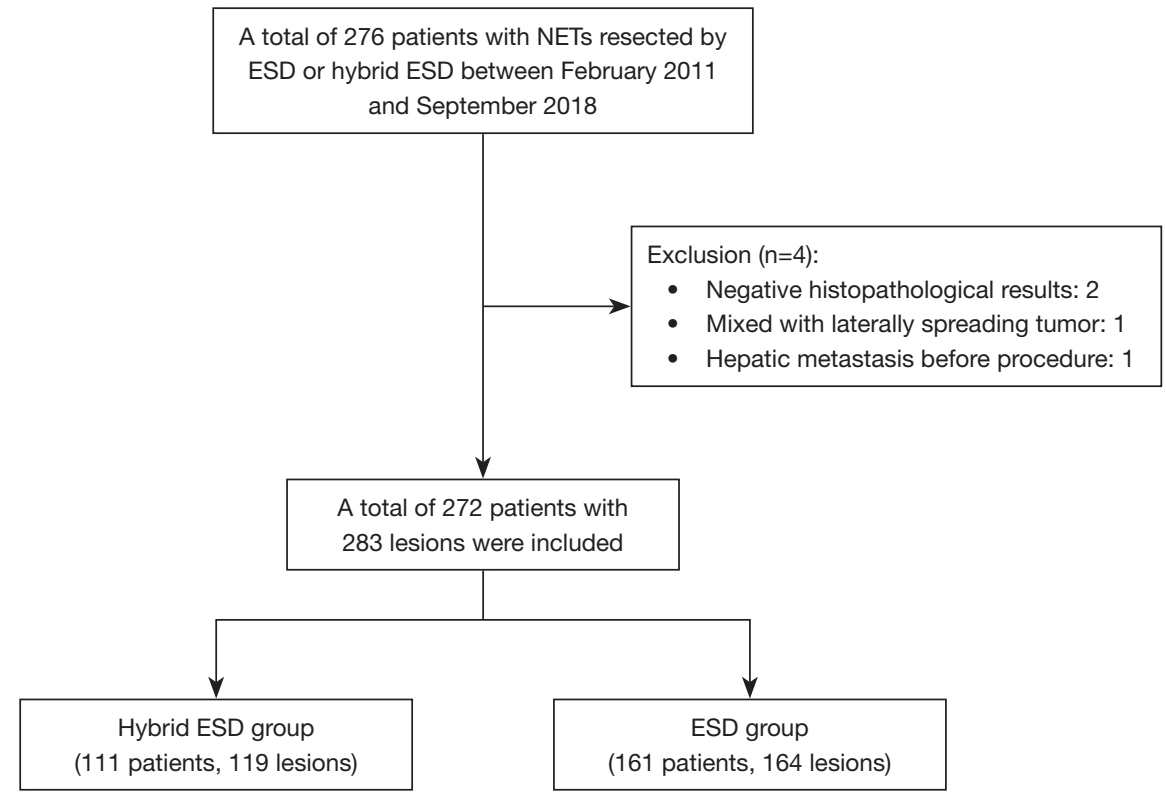

Figure 3 Flow chart of the patients and lesions enrolled in this study. ESD, endoscopic submucosal dissection. NET, neuroendocrine tumor.

successfully. Perforation did not occur in either group.

The overall median follow-up time was 31 months (range, 6 to 97 months). One patient in the ESD group experienced recurrence, which was confirmed by biopsy during the follow-up endoscopic examination 1 year after the ESD procedure. During the follow-up period, no evidence of recurrence or metastasis was detected in the other patients in either of the groups. Two patients who underwent ESD, and one patient who underwent hybrid ESD received additional surgery. The postoperative pathology results showed no positive tumor cells in two of the patients. Tumor cells were detected on the base of the ulcer in the other patient but without lymph nodes metastasis.

\section{Factors associated with short procedure time and incomplete resection}

The results of the univariate and multivariate analysis for shorter procedure time by logistic regression analysis are shown in Table 3. Although univariate analysis demonstrated that location, size, and procedure type were related to procedure time, the result of multivariate analysis showed that hybrid ESD (odds ratio, 2.661; $95 \%$ confidence interval, 1.599-4.427; $\mathrm{P}=0.000)$ and small lesion size (odds ratio, 4.285; 95\% confidence interval, 2.063-8.900; $\mathrm{P}=0.000)$ were strongly associated with shorter procedure time.

The outcomes of univariate analysis (Table 4) showed that histopathological grade was significantly different between complete and incomplete resection groups $(\mathrm{P}=0.004)$. Multivariate analysis including lesion location, size, histopathological grade and a layer of invasion showed that grade 2 (odds ratio, 3.587; 95\% confidence interval, $1.366-$ 9.418; $\mathrm{P}=0.010)$ was strongly associated with incomplete resection (Table 5).

\section{Discussion}

This was a comparative study that included a relatively large number of patients with rectal NETs at a tertiary center. The results of this study demonstrated ESD and hybrid ESD to each be an effective and safe procedure for treating rectal NET. Hybrid ESD can also achieve high rates of complete resection (94.1\%) and en bloc resection (99.2\%) without increases in adverse events and had a shorter procedure time compared with ESD. The higher histopathological grade was associated with incomplete resection.

Rectal NETs are usually asymptomatic and often discovered incidentally during a colonoscopy (14). With the development of high-resolution endoscopy, the detection of rectal NETs during endoscopic examinations has increased. 
Table 1 Baseline characteristics of patients and lesions

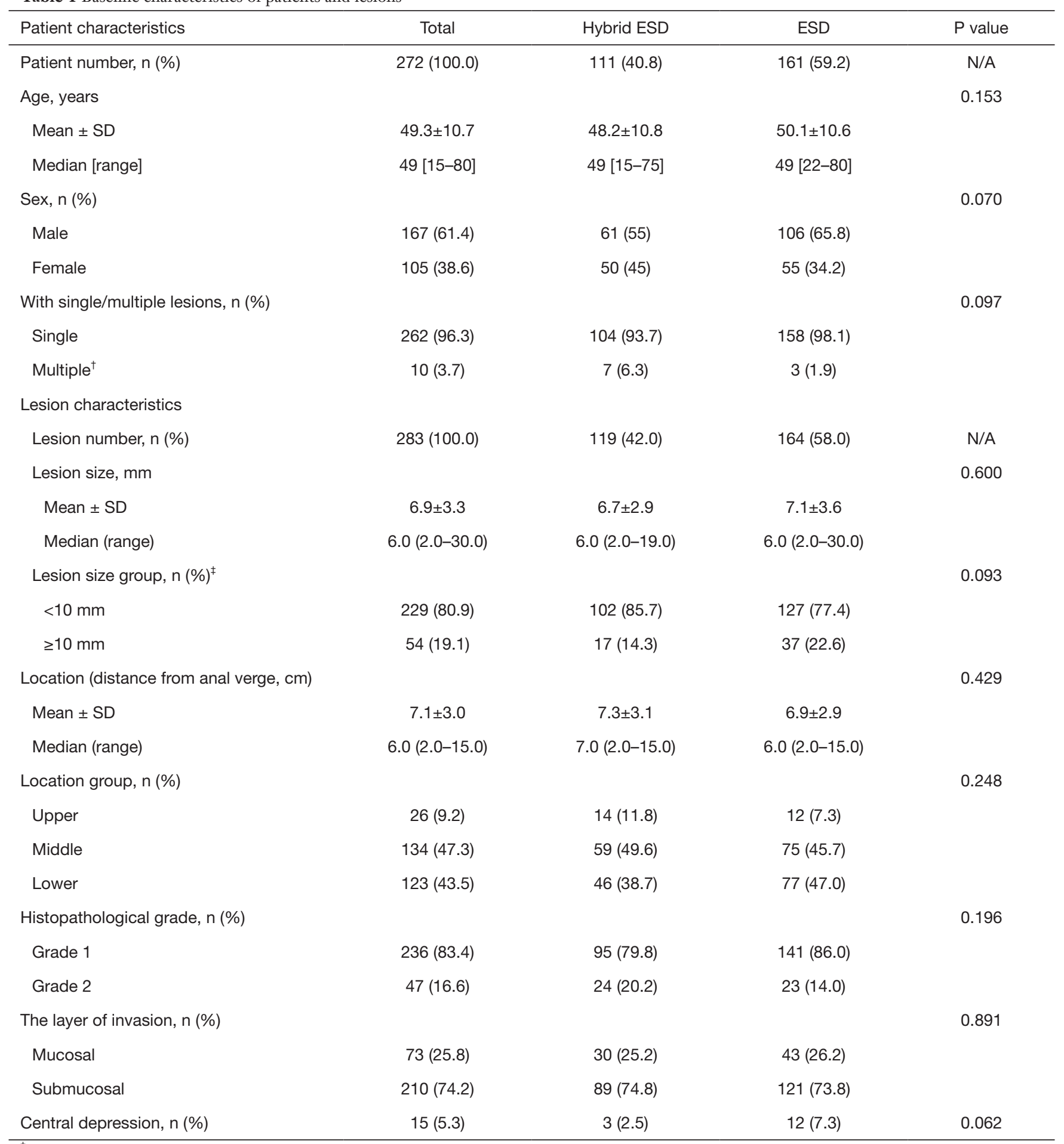

${ }^{\dagger}$, among the patients who had multiple lesions, one patient in the hybrid ESD group had three lesions, and the others each had two lesions; ${ }^{\ddagger}$, only one patient in the ESD group had a lesion with a diameter of $30 \mathrm{~mm}$, and the others had lesions less than $20 \mathrm{~mm}$ in diameter. ESD, endoscopic submucosal dissection; N/A, not available; SD, standard deviation. 
Table 2 Clinical outcomes of ESD and hybrid ESD

\begin{tabular}{|c|c|c|c|c|}
\hline Outcomes & Total $(n=283)$ & Hybrid ESD $(n=119)$ & ESD $(n=164)$ & $P$ value \\
\hline Mean \pm SD & $16.1 \pm 9.4$ & $13.2 \pm 8.3$ & $18.1 \pm 9.7$ & \\
\hline Median (range) & $13.0(4.0-56.0)$ & $10.0(4.0-48.0)$ & $16.0(5.0-56.0)$ & \\
\hline En bloc resection, $\mathrm{n}(\%)$ & $279(98.6)$ & $118(99.2)$ & $161(98.2)$ & 0.373 \\
\hline Histological margin involvement, $\mathrm{n}(\%)$ & & & & 0.597 \\
\hline Vertical & $17(6.0)$ & $6(5.0)$ & $11(6.7)$ & \\
\hline Lateral & $3(1.1)$ & $1(0.8)$ & $2(1.2)$ & \\
\hline Both vertical and lateral & $2(0.7)$ & $0(0.0)$ & $2(1.2)$ & \\
\hline Perforation & $0(0.0)$ & $0(0.0)$ & $0(0.0)$ & 1.000 \\
\hline Operation involving incomplete resection ${ }^{\dagger}, \mathrm{n}(\%)$ & $4(1.4)$ & $1(0.8)$ & $3(1.8)$ & 0.641 \\
\hline
\end{tabular}

${ }^{\dagger}$, because of positive resection margins, two patients in the ESD group and one patient in the hybrid ESD group underwent subsequent surgery, and one patient in the ESD group underwent additional ESD; *, P<0.05. ESD, endoscopic submucosal dissection; SD, standard deviation.

Table 3 Univariate and multivariate analysis for a short procedure time ( $<13$ minutes)

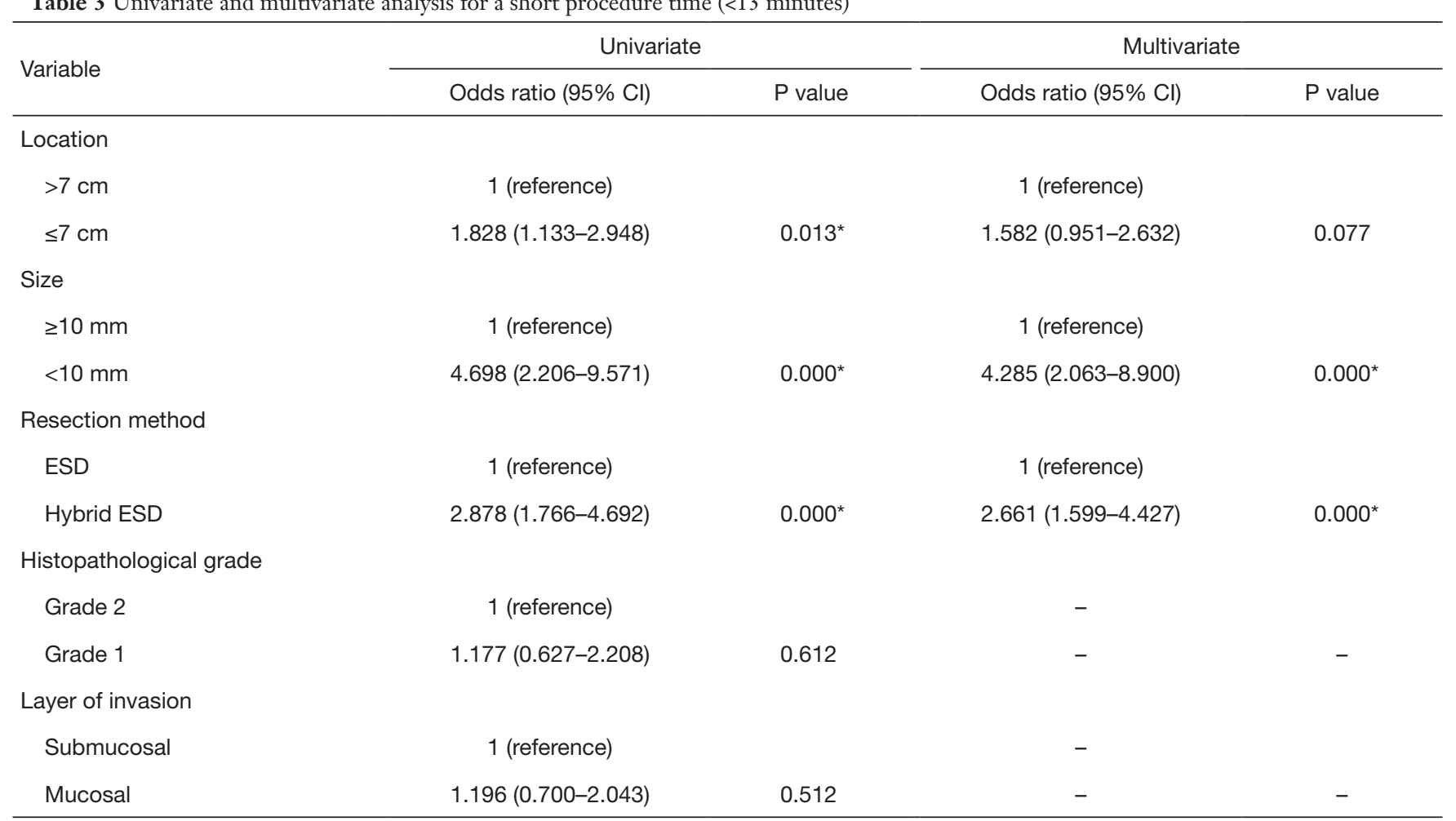

*, $\mathrm{P}<0.05$. ESD, endoscopic submucosal dissection; $\mathrm{Cl}$, confidence interval. 
Table 4 Univariate analysis for incomplete resection

\begin{tabular}{|c|c|c|c|}
\hline Variables & Complete resection $(n=261)$ & Incomplete resection $(\mathrm{n}=22)$ & $P$ value \\
\hline Mean \pm SD & $7.1 \pm 2.9$ & $6.5 \pm 3.3$ & \\
\hline Median (range) & $6.0(2.0-15.0)$ & $5.0(2.0-15.0)$ & \\
\hline Location group, n (\%) & & & 0.153 \\
\hline$>7 \mathrm{~cm}$ & $112(42.9)$ & $6(27.3)$ & \\
\hline Lesion size group, n (\%) & & & 0.193 \\
\hline$<10 \mathrm{~mm}$ & $214(82.0)$ & $15(68.2)$ & \\
\hline$\geq 10 \mathrm{~mm}$ & $47(18.0)$ & 7 (31.8) & \\
\hline Central depression, n (\%) & $13(5.0)$ & $2(9.1)$ & 0.741 \\
\hline Procedure types, n (\%) & & & 0.311 \\
\hline Hybrid ESD & $112(42.9)$ & $7(31.8)$ & \\
\hline ESD & $149(57.1)$ & $15(68.2)$ & \\
\hline Procedure time, min (mean $\pm S D$ ) & $15.9 \pm 9.4$ & $17.2 \pm 9.1$ & 0.372 \\
\hline En bloc resection, $\mathrm{n}(\%)$ & 258 (98.9) & $21(95.5)$ & 0.722 \\
\hline
\end{tabular}

*, $\mathrm{P}<0.05$. ESD, endoscopic submucosal dissection; SD, standard deviation; M, mucosal; SM, submucosal.

It has been reported that the incidence of rectal NETs detected during a screening colonoscopy is $0.17 \%(15)$. A better prognosis can be achieved if rectal NETs are treated at an early stage (1), and ER, such as EMR, modified EMR, and ESD, is an effective modality for treating rectal NETs with relatively little trauma (16). Hybrid ESD was shown to be an effective and safe modality for treating colorectal laterally spreading tumor with a procedure time that was shorter in length than that of ESD and comparable rates of en bloc resection and adverse events (11), but its use for rectal NETs still needs to be studied.

The results of this study show that the application of hybrid ESD is feasible and practical for the ER of rectal NETs. First, during the hybrid ESD procedure, after the circumferential incision and a certain amount of submucosal dissection, the remainder of the lesion could feasibly be resected completely by snaring, which is easy for endoscopists because snare resection techniques are used in the EMR procedure. Second, hybrid ESD can also achieve high rates of complete and en bloc resection compared with ESD. The rate of en bloc resection in this study was higher than that in a previous study of hybrid ESD for colorectal laterally spreading tumors $(10,11,17)$, which might be due to the relatively small size of the rectal NET lesions. The complete resection rate of hybrid ESD in this study was similar to that of ESD and higher than that of conventional EMR reported in a previous study (18). Moreover, hybrid ESD can serve as an alternative method in some situations when ESD is difficult to perform, such as in cases of poor patient tolerance, easy bleeding, or lesions that are difficult to access.

Previous studies showed that lesion size was a significant prognostic factor for rectal NETs, as well as other factors such as invasion depth, histological grade, and central depression $(5,6,19)$. Lesions less than $10 \mathrm{~mm}$ in size carry a low risk of metastasis and can be completely resected endoscopically. However, those between 10 and $20 \mathrm{~mm}$ in size remain controversial. Recent studies have shown oncological safety comparable to that of local surgical excision for lesions $20 \mathrm{~mm}$ or smaller in diameter without adverse features and treated with ER (20). In the present study, $80.9 \%$ of the tumors were less than $10 \mathrm{~mm}$ in size, 
Table 5 Multivariate analysis for incomplete resection

\begin{tabular}{lccc}
\hline Variables & OR & $95 \% \mathrm{Cl}$ & P value \\
\hline $\begin{array}{l}\text { Location group } \\
\leq 7 \mathrm{~cm}\end{array}$ & 1 (reference) & & \\
$>7 \mathrm{~cm}$ & 0.538 & $0.199-1.455$ & 0.222 \\
Lesion size group & & & \\
$<10 \mathrm{~mm}$ & 1 (reference) & & \\
$\geq 10 \mathrm{~mm}$ & 1.312 & $0.472-3.652$ & 0.603 \\
Layer of invasion & & & \\
Mucosal & 1 (reference) & & \\
Submucosal & 3.472 & $0.778-15.503$ & 0.103 \\
Histopathological grade & & \\
Grade 1 & 1 (reference) & & \\
Grade 2 & 3.587 & $1.366-9.418$ & $0.010^{*}$ \\
\hline
\end{tabular}

*, $\mathrm{P}<0.05$. OR, odds ratio; $\mathrm{Cl}$, confidence interval.

whereas $19.1 \%$ of the tumors larger than $10 \mathrm{~mm}$. More data are needed to confirm the safety of ESD and hybrid ESD for tumors large than $10 \mathrm{~mm}$.

The management of incomplete rectal NET resection is still controversial. A positive resection margin is not a completely satisfactory predictive factor and could result in unnecessary (and potentially damaging) surgical overtreatment. The destructive impact of cauterization on nearby tumor cells during ER may have a sterilizing effect on the resection site (21), which has been shown by the follow-up data of studies including patients with incompletely resected rectal NETs $(18,22)$. However, regarding the slow progression and limited information on the long-term outcomes and poor prognosis of metastasis and recurrence of rectal NETs, management strategies should be comprehensive and account for the wishes of the patients. Most of the patients in this study with incomplete resection chose to be followed with regular endoscopic and imaging examinations. The follow-up results showed no recurrence in these patients, but further follow-up data are still needed. Only two patients who had received ESD and one patient who had received hybrid ESD underwent additional surgery, but the postoperative pathology results showed no positive tumor cells in two of the patients. One patient in the ESD group chose to undergo an additional ESD procedure as a rescue treatment and achieved complete resection, which indicated that additional ESD might be an alternative for experienced endoscopists.

This study has some limitations. First, due to its retrospective nature and single tertiary center, it may include selection bias. Second, bearing in mind the characteristics of slow-growing rectal NETs and that recurrence may occur several years after patients are first treated, the follow-up period of some patients in this study, especially those who experienced incomplete resection, is relatively insufficient. The clinical outcomes of disease-free survival or time to recur are not evaluated due to the lower recur rate at present. There is a need for further prospective studies with a long-term follow-up period to further examine these issues. Third, the complete resection rate for rectal NETs remains unsatisfactory, and new techniques are urgently needed.

Further studies on the submucosal tunneling technique $(23,24)$, endoscopic full-thickness resection (EFTR) $(25,26)$ are needed to improve the complete resection. Additionally, the outcomes of other resection methods such as conventional EMR or modified EMR was not compared in this study. To provide more efficient and safer treatments, the selection of different resection techniques based on each patient's condition and lesion characteristics may be a better management strategy for patients with rectal NETs.

To conclude, both ESD and hybrid ESD are effective and safe forms of treatment for rectal NET. For rectal NET patients, hybrid ESD provides an alternative treatment option. Techniques must be further developed to achieve improvements in the rate of complete resection, especially with tumors with a higher histopathological grade.

\section{Acknowledgments}

We thank all colleagues for their support of this work. We thank the National Key R\&D Program of China for funding this project.

Funding: The study was funded by the National Key R\&D Program of China (No. 2016YFC1303601).

\section{Footnote}

Conflicts of Interest: The authors have no conflicts of interest to declare.

Ethical Statement: The authors are accountable for all aspects of the work in ensuring that questions related to the accuracy or integrity of any part of the work are appropriately investigated and resolved. Approval was 
granted by the Ethics Committee of the Institutional Review Board of Chinese PLA General Hospital (No. S2017-010-02) complying with the Declaration of Helsinki.

Open Access Statement: This is an Open Access article distributed in accordance with the Creative Commons Attribution-NonCommercial-NoDerivs 4.0 International License (CC BY-NC-ND 4.0), which permits the noncommercial replication and distribution of the article with the strict proviso that no changes or edits are made and the original work is properly cited (including links to both the formal publication through the relevant DOI and the license). See: https://creativecommons.org/licenses/by-nc-nd/4.0/.

\section{References}

1. Dasari A, Shen C, Halperin D, et al. Trends in the Incidence, Prevalence, and Survival Outcomes in Patients With Neuroendocrine Tumors in the United States. JAMA Oncol 2017;3:1335-42.

2. Hallet J, Law CH, Cukier M, et al. Exploring the rising incidence of neuroendocrine tumors: a population-based analysis of epidemiology, metastatic presentation, and outcomes. Cancer 2015;121:589-97.

3. Fraenkel M, Kim M, Faggiano A, et al. Incidence of gastroenteropancreatic neuroendocrine tumours: a systematic review of the literature. Endocr Relat Cancer 2014;21:R153-63.

4. de Mestier L, Brixi H, Gincul R, et al. Updating the management of patients with rectal neuroendocrine tumors. Endoscopy 2013;45:1039-46.

5. Konishi T, Watanabe T, Kishimoto J, et al. Prognosis and risk factors of metastasis in colorectal carcinoids: results of a nationwide registry over 15 years. Gut 2007;56:863-8.

6. Caplin M, Sundin A, Nillson O, et al. ENETS Consensus Guidelines for the Management of Patients with Digestive Neuroendocrine Neoplasms: Colorectal Neuroendocrine Neoplasms. Neuroendocrinology 2012;95:88-97.

7. Zhong DD, Shao LM, Cai JT. Endoscopic mucosal resection vs endoscopic submucosal dissection for rectal carcinoid tumours: a systematic review and meta-analysis. Colorectal Dis 2013;15:283-91.

8. Zhou X, Xie H, Xie L, et al. Endoscopic resection therapies for rectal neuroendocrine tumors: a systematic review and meta-analysis. J gastroenterol hepatol. 2014;29:259-68.

9. Chablaney S, Zator ZA, Kumta NA. Diagnosis and management of rectal neuroendocrine tumors. Clin Endosc 2017;50:530-6.

10. Byeon JS, Yang DH, Kim KJ, et al. Endoscopic submucosal dissection with or without snaring for colorectal neoplasms. Gastrointest Endosc 2011;74:1075-83.

11. Bae JH, Yang DH, Lee S, et al. Optimized hybrid endoscopic submucosal dissection for colorectal tumors: a randomized controlled trial. Gastrointest Endosc 2016;83:584-92.

12. Bosman FT, Carneiro F, Hruban RH, et al. Who classification of tumours of the digestive system. Lyon: IARC, 2010.

13. Milano RV, Viale E, Bartel MJ, et al. Resection outcomes and recurrence rates of endoscopic submucosal dissection (esd) and hybrid esd for colorectal tumors in a single italian center. Surg Endosc 2018;32:2328-39.

14. Hassan MM, Phan A, Li D, et al. Risk factors associated with neuroendocrine tumors: a u.s.-based case-control study. Int J Cancer 2008;123:867-73.

15. Jung YS, Yun KE, Chang Y, et al. Risk factors associated with rectal neuroendocrine tumors: a cross-sectional study. Cancer Epidemiol Biomarkers Prev 2014;23:1406-13.

16. Park HW, Byeon JS, Park YS, et al. Endoscopic submucosal dissection for treatment of rectal carcinoid tumors. Gastrointest Endosc 2010;72:143-9.

17. Kim YJ, Kim ES, Cho KB, et al. Comparison of clinical outcomes among different endoscopic resection methods for treating colorectal neoplasia. Dig Dis Sci 2013;58:1727-36.

18. Kaneko H, Hirasawa K, Koh R, et al. Treatment outcomes of endoscopic resection for rectal carcinoid tumors: an analysis of the resectability and long-term results from 46 consecutive cases. Scand J Gastroenterol 2016;51:1489-94.

19. McDermott FD, Heeney A, Courtney D, et al. Rectal carcinoids: a systematic review. Surg Endosc 2014;28:2020-6.

20. Sui Q, Lin J, Peng J, et al. Local surgical excision versus endoscopic resection for rectal carcinoid: a meta-analysis. J Cancer 2017;8:3969-74.

21. Park CH, Cheon JH, Kim JO, et al. Criteria for decision making after endoscopic resection of well-differentiated rectal carcinoids with regard to potential lymphatic spread. Endoscopy 2011;43:790-5.

22. Choi CW, Park SB, Kang DH, et al. The clinical outcomes and risk factors associated with incomplete endoscopic resection of rectal carcinoid tumor. Surg Endosc 2017;31:5006-11. 
23. Linghu E, Feng X, Wang X, et al. Endoscopic submucosal tunnel dissection for large esophageal neoplastic lesions. Endoscopy 2013;45:60-2.

24. Kobara H, Mori H, Chei L, et al. The advantage of an endoscopic submucosal tunneling technique for rectal carcinoid tumors. Gut Liver 2017;11:735-7.

25. Mori H, Kobara H, Nishiyama N, et al. Current status and future perspectives of endoscopic full-thickness resection. Dig Endosc 2018;30 suppl 1:25-31.

26. Cai M, Zhou P, Lourenço LC, et al. Endoscopic Fullthickness Resection (EFTR) for Gastrointestinal Subepithelial Tumors. Gastrointest Endosc Clin N Am 2016;26:283-95.
Cite this article as: Wang XY, Chai NL, Linghu EQ, Li HK, Zhai YQ, Feng XX, Zhang WG, Zou JL, Li LS, Xiang JY. Efficacy and safety of hybrid endoscopic submucosal dissection compared with endoscopic submucosal dissection for rectal neuroendocrine tumors and risk factors associated with incomplete endoscopic resection. Ann Transl Med 2020;8(6):368. doi: 10.21037/atm.2020.02.25 